\title{
"Ich werde hier sagen, was ich will!" Sprachkritische Betrachtungen und Kritik an der Sprachkritik im Kontext des aktuellen Flüchtlingsdiskurses
}

\author{
"Ich werde hier sagen, was ich will!" \\ Critical Reflections on Uses of Language in the Context of Current \\ Debates About Refugees in Germany
}

Markéta Ederová

\begin{abstract}
This contribution deals with critical consciousness and reflection on uses of language. In the recent context of political developments in Germany there is an intensified discussion about proper application of terms, the power of words and the importance of intellectual reflections on these changements. In 2015 the German language society GfDS declared the word Flüchtling (means refugee) as "Word of the Year", therefore it's probably the best example to undertake such analysis. The term "Flüchtling" exactly is situated on the edge between sensitivity in using language, words, on the one side - and on the other the speechlessness in order to "political correctness". The broader framework of the article discusses then the critical action "Unwort des Jahres" (means most inappropriate word used for special problems) - worth while a liguistic based analysis. The outlinig questions here have to be: is there a guide line, a direction in chosing such "Unworte" and words next to them? Are there linguistic strategies in order to construction of terms, words and its using?
\end{abstract}

\section{Keywords}

language criticism, "Word of the Year", political correctness, discourse analysis, topos 
„Die ,Flüchtlingskrise“ ist auch eine Sprachkrise“, verkündete lapidar die Überschrift zu einer der zahlreichen sprachreflexiven Betrachtungen und Exkursen, die in den letzten Jahren in den Zeitungen ihren festen Platz fanden. ${ }^{1}$ Die Sprache ist wieder verstärkt in den Fokus öffentlicher Aufmerksamkeit gerückt - in den Schlagzeilen großer Zeitungen werden Sprachwissenschaftler zitiert, Präfixe und Suffixe auch außerhalb der Vorlesungssäle abgehandelt und die Wortbildung steigt zum Gegenstand hitziger Auseinandersetzungen in sozialen Netzwerken auf. Die konkreten Auslöser der sprachkritischen bzw. sprachreflexiven Debatten, auf die weiter unten eingegangen wird, machen den engen Zusammenhang mit der gesellschaftlichen Entwicklung und den aktuellen politischen Herausforderungen deutlich.

Die Handlungspotenz der Sprache wurde natürlich nicht erst jetzt erkannt. Während es aber die meiste Zeit über vor allem den Schriftstellern, Philosophen und Sprachwissenschaftlern vorbehalten blieb, über die Sprache und ihre Macht nachzudenken, haben etwa seit den 1970er Jahren sprachkritische Reflexionen auch in der Politik Fuß gefasst. In der Studentenbewegung von 68 wurde das immense Manipulationspotential der Sprache explizit thematisiert und die Sprache als zentrales Herrschaftsmittel „entlarvt“. Die griffige Formel Begriffe besetzen stammt zwar aus dem politischen Lager der CDU, konkret aus der Feder ihres Generalsekretärs Kurt Biedenkopf, ist aber als eine allgemein geteilte, aus diesen sprachkritischen Diskussionen resultierende Erkenntnis zu deuten.

„Revolutionen finden heute auf andere Weise statt. Statt der Gebäude der Regierungen werden die Begriffe besetzt, mit denen sie regiert, die Begriffe, mit denen wir unsere staatliche Ordnung, unsere Rechte und Pflichten und unsere Institutionen beschreiben. [...] Wir erleben heute eine Revolution, die sich nicht der Besetzung der Produktionsmittel, sondern der Besetzung der Begriffe bedient." 2

Eine weitere Station auf dem Weg der Sprache aus dem Elfenbeinturm ${ }^{3}$ in das reale Leben markiert der Germanistentag im Jahr 1984, in dessen vierter Sektion die Generalsekretäre der beiden großen Parteien CDU und SPD Vorträge hielten. ${ }^{4}$ Es beteiligen sich also immer mehr Akteure am sprachkritischen Diskurs, Politiker und Journalisten aber immerhin als diejenigen, die beruflich mit der Sprache zu tun haben. Neuerdings kommt aber eine neue Dimension hinzu: Die Stimme der Öffentlichkeit, deren sprachliches Bewusstsein wohl in den meisten Fällen - soweit sich aus den Äußerungen darauf schließen lässt - sehr rudi-

1 http://www.derwesten.de/politik/welle-lawine-fluechtlingskrise-ist-auch-eine-sprachkrise-id11278969. html, letzter Zugriff am 9. 3. 2017.

2 Zitiert nach KILIAN, Jörg (Hrsg.): Sprache und Politik. Deutsch im demokratischen Staat. Mannheim: Dudenverl., 2005, S. 183.

3 Der Auszug aus dem Elfenbeinturm beginnt - Bericht über den Germanistentag in Passau in der ZEIT, online verfügbar unter http://www.zeit.de/1984/44/liebenswuerdig-harmonisch-und-ein-bisschen-politisch, letzter Zugriff am 9. 3. 2017.

4 GEISSLER, Heiner: Sprache und Politik. Kampf um Begriffe. Nachzulesen in: STÖTZEL, Georg (Hrsg.): Germanistik - Forschungsstand und Perspektiven. Vorträge des Deutschen Germanistentages 1984. Berlin: de Gruyter, 1984, S. 222-230. GLOTZ, Peter: Die Rückkehr der Mythen in die Sprache der Politik. Ebd., S. 231-244. Kommentiert wurden diese Beiträge von einem der Hauptvertretern der Politolinguistik Walther DIECKMANN: Herrschaft durch Sprache durch Herrschaft über Begriffe. Ebd., S. 245-252. 
mentär, punktuell und unsystematisch entwickelt und eine Art Freizeitbeschäftigung ist, kein spezieller Gegenstand, sondern ein Thema unter unzähligen anderen, zu denen man seinen Kommentar hinzufügen zu müssen glaubt. Diese „Akteursgruppe“ war vermutlich - in welchem Ausmaß auch immer - schon immer Teil des sprachkritischen Diskurses, da uns der Zugriff auf diesen aber nur mittels geschriebener Texte (also Zeitungen - und hier auch nur ausschnittsweise: die digitalisierten) möglich ist, kann man erst für den Zeitraum der letzten Jahre von Relevanz dieser „Stimme“ ausgehen. Die Leserkommentare in den Onlineausgaben der Zeitungen, Beiträge in verschiedenen Internetforen, Twittermeldungen usw. machen es also möglich, die sprachkritische Debatte auch auf dieser Ebene zu verfolgen und den verschiedenen laienlinguistischen Auffassungen von Sprache in der Bevölkerung nachzuspüren. Mit „laienlinguistisch“ sind sowohl die Äußerungen in der Presse als auch in den Internet-Diskussionsrunden gemeint, natürlich mit den stets zu beachtenden Unterschieden von schriftlich/mündlich, gründlich recherchiert und fachlich informiert/vorwiegend von linguistischen Bauchgefühlen dominiert, das Gebot bzw. den Ehrenkodex der politischen Korrektheit respektierend/sich nicht selten Stammtischparolen bedienend. Allerdings lassen sich - mit der anhand des oben Gesagten zu erwartenden Gewichtung - bestimmte grundlegende Positionen und Einstellungen zur Sprache ausmachen. Dies versucht der Aufsatz aufzuzeigen, indem er zunächst kurz auf die einzelnen Stränge des aktuellen sprachkritischen Diskurses eingeht und anschließend die medialen Reaktionen analysiert. Die zentrale Fragestellung dabei ist: Sind die Erkenntnisse der Sprachwissenschaft, genau gesagt ihrer relativ neuen Disziplinen wie Politolinguistik oder Diskursanalyse im Alltag angekommen? Erreicht das sprachkonstruktivistische Paradigma, das nicht nur die Linguistik, sondern auch andere Wissenschaften wie Soziologie oder Humangeographie dominiert, das Bewusstsein eines „Normalsprechers“, prägt es seine Vorstellungen von der Sprache bzw. vom Verhältnis von Wirklichkeit und Sprache? Es wird an die einzelnen Äußerungen mit den Methoden der linguistischen Diskursanalyse herangegangen und nach sich wiederholenden Gedankenfiguren (Topoi) gesucht, die auf bestimmte repräsentative Denkmuster schließen lassen bzw. besonders gut geeignet sind, das „in einer Zeit verbreitetes kollektives, gesellschaftliches Wissen zu erfassen“5.

Als Einstieg eignet sich das Wortgefecht, das sich der CSU-Politiker Peter Ramsauer, die Generalsekretärin der Bayern-SPD Natascha Kohnen und der ehemalige BILD-Chefredakteur Hans Hermann Tiedje in der ARD-Talkshow Maischberger lieferten ${ }^{6}$, denn darin manifestieren sich auf sehr anschauliche Weise die beiden extremen Pole der Sprachauffassung, die sich leicht variierend im gesamten Diskurs wiederfinden. Hier wortgetreu und leicht gekürzt wiedergegeben:

Ramsauer: „Ich sage hier ganz ehrlich: das, was Mazedonien gemacht hat, was Ungarn schon gemacht hat, was irgendwo auch jetzt in ähnlicher Weise Österreich macht - das kann hundertmal EU-rechtlich Unrecht sein, das ist eine Art Notwehr [...].“

5 WENGELER, Martin: Topos und Diskurs - Möglichkeiten und Grenzen der topologischen Analyse gesellschaftlicher Debatten. In: WARNKE, Ingo H. (Hrsg.): Diskurslinguistik nach Foucault. Theorie und Gegenstände, Berlin: De Gruyter 2007, S. 165-186.

6 Hass auf Flüchtlinge, Regierung zerstritten: Spaltet Merkel das Land?, Sendung vom 24. Februar 2016. 
Kohnen: „Wenn Sie Begriffe wie Notwehr verwenden - wir sprechen immer noch von Menschen, und diese Begrifflichkeiten wie Notwehr führen ganz genau dazu, dass die Bevölkerung... im Prinzip ist es Enthemmung der politischen Sprache: Notwehr, Rechtsbruch - oder wie auch Ihr Vorsitzender Seehofer mal gesagt hat ,wir werden uns als CSU wehren bis zur letzten Patrone, damit es zu keiner Zuwanderung in die sozialen Sicherungssysteme kommt. ' Solche Begrifflichkeiten führen exakt dazu, dass es in der Bevölkerung auch zu so einer Enthemmung kommt. Und insofern bitte ich einfach wirklich schon mal auf die Sprache zu achten. Notwehr ist in dem Verhältnis mit den Flüchtlingen einfach keine Rhetorik. [...]

Tiedje: „Eingangs Folgendes: erstmal an Ihre Adresse: Wir brauchen hier keine Sprachpolizei. [...] Ich werde hier sagen, was ich will. Ich nenne es eine Katastrophe, ich nenne es eine Flut - ich nenne es einfach so, weil es so ist. Ich brauche jetzt keine Sprachreinigung, ich sollte es nicht so nennen, weil es Ihnen nicht gefällt? Also für die nächsten sechzig Minuten müssen Sie mit meiner Sprache auskommen."7

Es ist der Ausdruck „Notwehr“, der hier als Zündstoff funktioniert - ein schönes Beispiel der sprachlichen Strategie, die in der Politolinguistik als „parteiliches Prädizieren“ bezeichnet wird. ${ }^{8}$ Damit ist die Konkurrenz in der Bezeichnung eines meist umstrittenen Sachverhaltes gemeint, für den man mit Hilfe eines bestimmten Ausdrucks die eigene Deutung durchzusetzen versucht. Andere Beispiele wären Anschluss vs. Beitritt [der DDR zur BRD] oder multikulturelle Gesellschaft vs. Parallelgesellschaft. ${ }^{9}$ Die Reaktion von Natascha Kohnen ist eine sprachkritische: sie verweist auf das Handlungspotenzial der Sprache, warnt vor einer Enthemmung zunächst der Sprache und in Folge dann des Verhaltens und mahnt zur Selbstdisziplin, wofür sie sich das Etikett einer Sprachpolizistin einhandelt.

Mit der Enthemmung wird ein Begriff aus der Psychologie politisch umgedeutet. Die typischen Verwendungsbeispiele aus der Alltagssprache, die im DUDEN ${ }^{10}$ angeführt werden, legen nahe, dass die Hemmung nicht vorrangig etwas eindeutig Negatives ist (im Sinne von „Hindernis, das es zu beseitigen gilt“), sondern eher Schutz bzw. eine Art moralische Handbremse. Hemmung ist nach der DUDEN-Definition ${ }^{11}$ also „etwas, was jemanden in seinem Inneren (aufgrund einer bestimmten ethischen Norm) daran hindert, etwas Bestimmtes zu tun“ - und genau dies ist die Position der Sprachbewussten, die Wert auf sprachliche Selbstdisziplinierung und Behutsamkeit, auf Sensibilisierung legen - sie sind der Überzeugung, dass sprachliche Leichtfertigkeiten und Zügellosigkeit (eben ähnlich wie Alkohol und Drogen) schwerwiegende Konsequenzen haben können. Aber Hemmung hat noch eine andere Bedeutungsnuance, nämlich ,jemanden in der

7 Transkript angefertigt von der Verfasserin dieses Beitrags.

8 Die Unterteilung der „semantischen Kämpfe“ in der Politik v.a. bei Josef KLEIN: Kann man „Begriffe besetzen“? Zur linguistischen Differenzierung einer plakativen politischen Metapher. In: LIEDTKE, Frank/ WENGELER, Martin/BÖKE, Karin (Hrsg.): Begriffe besetzen. Strategien des Sprachgebrauchs in der Politik. Opladen: Westdt. Verl., 1991, S. 44-69, hier zitiert nach NIEHR, Thomas: Einführung in die Politolinguistik. Gegenstände und Methoden. Göttingen: Vandenhoeck \& Ruprecht, 2014, S. 89.

9 KILIAN [Anm. 2], S. 189.

10 http://www.duden.de/rechtschreibung/enthemmen\#b2-Bedeutung-1

11 http://www.duden.de/rechtschreibung/Hemmung 
Entfaltung seiner Persönlichkeit sehr behindernde, beeinträchtigende (innere Unsicherheit)“. Dieser Aspekt und die negativen Folgen einer „gehemmten“ (korrekten) Sprache werden von den Gegnern der Sprachsensibilisierung hervorgehoben - ihr Pochen auf die Meinungs- und Redefreiheit verdichtet sich eben in dem knappen und trotzigen Ausruf „Ich werde hier sagen, was ich will!“”

Diese Positionen durchziehen in verschiedenen Variationen den gesamten sprachkritischen Diskurs - und hinter der Unversöhnlichkeit der Handlungsansätze stecken zwei diametral unterschiedliche Auffassungen von Sprache: Die eine Position räumt der Sprache immense Wichtigkeit bei der Wahrnehmung der Welt ein, bei der Entstehung von Weltbildern, kurz bei der Konstitution der Wirklichkeit. Man könnte hier vom Sprachkonstruktivismus reden - und es verwundert nicht, dass in diesem Meinungslager vorwiegend (aber nicht ausschließlich) diejenigen vertreten sind, die mit der Sprache viel und vor allem beruflich zu tun haben, also Sprachwissenschaftler und Journalisten. Der zentrale Topos ist das metaphorische Konzept „Sprache ist Macht“ bzw. „Sprache ist eine Waffe“ - und die dadurch freigesetzte argumentative Strategie verläuft wie folgt: Sprache ist eine Waffe, mit der man eben sehr wirksam „zuschlagen“ kann („(Zu-)Schlagargument") und deshalb vorsichtig umgehen muss. Eine unüberlegte Äußerung kann „verbaler Brandstiftung“ gleichkommen, man kann „mit seinen Worten Öl ins Feuer gieBen“ oder „mit Worten aufmuskeln“. Der sorgenfreie, entspannte, lockere Umgang mit der Sprache wird durchwegs negativ konnotiert - eben als Leichtsinnigkeit und Spiel mit dem Feuer. Der angemessene Sprachgebrauch hingegen besteht in der selbstauferlegten Hemmung und in der Einsicht, dass man eben nicht alles sagen darf - vor allem im Kontext der Lawine-Äußerung und der Flüchtlingsdebatte häufen sich Empfehlungen, „die Debatte besonnen zu führen“, „kein Katastrophenvokabular zu verwenden“. Die im Kontext sprachkritischer Debatten medial sehr präsente Linguistin Wehling fordert ganz entschieden: „Streichen Sie das Wort Flüchtling aus Ihrem Vokabular! Sie richten damit Schaden an." ${ }^{12}$ Angesichts der potentiellen Gefahr und der Notwendigkeit zur Selbstdisziplin kann aber nicht das (potentiell unterentwickelte) Sprachgefühl jedes Einzelnen die Richtinstanz sein, sondern es muss ständig an einer „Sensibilisierung“ gearbeitet werden. In diesem Kontext ist beispielsweise auch die sprachkritische Aktion „Unwort des Jahres“ zu sehen, auf die weiter unten ausführlicher eingegangen wird. Die Sensibilisierung sei vor allem im Bereich des metaphorischen Sprechens notwendig, denn wenn auffällige "Signalwörter“ fehlen, werden falsche Bilder auf unkontrollierte Weise und unbewusst durch die Sprache transportiert.

Die entgegengesetzte Diskursposition sieht derartige begriffliche Auseinandersetzungen als vollkommen überflüssig an und ist der Überzeugung, dass die Macht der Sprache überschätzt würde und die Sprachkritik unnützer Zeitvertreib sei. Die Sprache in dieser Auffassung ist kein potentiell gefährliches Instrument zur diskursiven Konstruktion der Wirklichkeit, sondern ein „Gerede“, anstatt dessen „Taten“ gefordert werden. Dahinter verbirgt sich ein essentialistisches Weltbild, ein fester Glaube an außersprachliche Realität, die handfest und objektiv gegeben ist und die primäre Bezugsgröße. Sprache hingegen

12 http://www.sueddeutsche.de/kultur/sprache-in-der-fluechtlingsdebatte-das-wort-fluechtling-richtet-schaden-an-1.2864820, letzter Zugriff am 14. 3. 2017. 
ist etwas Sekundäres, die ihr vorgängige und von ihr unabhängige Wirklichkeit nur Beschreibendes und Etikettierendes und daher im Grunde Überflüssiges. Im Folgenden eine kleine, aber durchaus repräsentative Auswahl:

„Ich würde mich mehr darüber freuen, wenn die Gerechtigkeits-Energie sich dafür auf würdigere Ziele richtete, auf eine menschenwürdige Behandlung von Frauen, Männern und insbesondere Kindern, die ihre Heimat aus welchen Gründen auch immer und mit welcher Wortbildung auch immer verlassen haben." ${ }^{13}$

„Man kann alles hineininterpretieren [...] Anstatt sich über Wörter den Kopf zu zerbrechen, sollten diese Experten ihr Wissen vielleicht besser in Sprachkurse und Bildungsangebote für die Flüchtlinge investieren." 14

„Versuchen Sie es einmal mit ehrlicher Arbeit, nicht mit dem an den Haaren Herbeiziehen neuer Definitionen und Umdeutungen eindeutig belegter Worte." 15

Und weil es eben objektiv existierende Wirklichkeit und Wahrheit gibt, reicht es, sie zu erkennen und im zweiten Schritt dann zu benennen. „Ich nenne es so, weil es so ist!“ Dies ist auch die argumentative Grundlage des ebenfalls in allen Teildiskursen präsenten Topos der „Redefreiheit“. Diese Sprachauffassung ist relativ eindeutig an die dritte Akteursgruppe gebunden (die Leser und „User“, die ihre Meinungen in den Online-Kommentarbereichen der Zeitungen und in sozialen Netzwerken kundtun), deren Stimme vielleicht aus der traditionellen Perspektive als weniger „offiziell“ gelten könnte, angesichts der veränderten Bedingungen des Veröffentlichens, d. h. Öffentlich-Werdens aber zweifellos auch ins Gewicht fällt. Die von den Gegnern im Schild geführte Notwendigkeit des sensiblen Sprachgebrauchs wird massiv in Zweifel gezogen bzw. ad absurdum geführt und so dessen Unsinnigkeit manifestiert: vor lauter Nachdenken, welches Wort man noch sagen darf und welches nicht, verstummt man. Die Erkenntnis, dass es unmöglich ist, über die meisten kontroversen Ereignisse und Sachverhalte neutral oder möglichst wertfrei zu sprechen, kann also zum einen in den „differenzierten Sprachgebrauch“, der aber zuweilen eigenartige Blüten treiben kann, zum anderen in die Sprachlosigkeit führen, die aber einer Kapitulation mit verheerenden Folgen gleichkommt. Dieser Topos der Sprachlosigkeit taucht immer wieder auf, zuweilen auch außerhalb der Twitterforen. Beispielsweise in der FAZ heißt es über die „sprachlichen Entgleisungen“ von Joachim Herrmann, Ranga Yogeshwar und Simone Peter in der Sendung Hart aber fair (näher dazu weiter unten): „So demonstrierten alle drei ihre persönlichen Schwierigkeiten mit dem richtigen Sprachgebrauch. Vielleicht diskutieren sie in Zukunft besser auf Latein, um das zu vermeiden." 16

13 Leserkommentare zu: http://www.sprachlog.de/2012/12/01/fluechtlinge-und-gefluechtete/, letzter Zugriff am 10. 3. 2017.

14 Leserkommentare zu: http://www.zeit.de/gesellschaft/zeitgeschehen/2015-12/fluechtlinge-wort-desjahres\#comments, letzter Zugriff am 10. 3. 2017.

15 Leserkommentare zu: http://derstandard.at/2000022449906/Asylanten-Fluechtlinge-Refugees-und-Vertriebene-eineSprachkritik, letzter Zugriff am 10. 3. 2017.

16 http://www.faz.net/aktuell/feuilleton/tv-kritik-hart-aber-fair-wir-koennen-das-schaffen-13779138.html, letzter Zugriff am 10. 3. 2017. 
Auf der einen Seite positionieren sich also die sprachbewussten „Sensibilisierer“, auf der anderen die selbstbewussten „Tatkräftigen“. Diese stören sich am meisten an dem moralischen Impetus, der mit der Forderung nach Sensibilisierung einhergeht - ganz explizit kommt es in der Kinderbuch-Debatte zum Ausdruck, denn die „Mission“ besteht ja eben darin, den Kindern durch die richtige Sprache das richtige Denken beizubringen. Der gleiche erzieherische Auftrag wird hinter der Forderung nach bewusstem Umgang mit Sprache vermutet, sehr oft tauchen in den Kommentaren Stichworte auf wie „Volkserziehung“ und „Volkspädagogik“, gepaart mit dem Vorwurf, die Sprachsensibilisierer würden sich moralisch überlegen fühlen. Dieser Topos des arroganten „selbsternannten Wächters“ hat zwei Komponenten - zum einen leitet die Metapher der Überwachung direkt zu dem noch extremer zugespitzten zentralen Topos der „Sprachpolizei“ über, zum anderen wird hier die Frage nach Autorität aufgeworfen: den Experten wird mangelndes Wissen vorgeworfen und ihr Expertentum abgesprochen, bestenfalls werden sie ignoriert und die Debatten werden von linguistischen Bauchgefühlen dominiert. Dies ist bei allen hier ausgewählten Teildiskursen auffällig - die Suche nach sachlichen Argumenten, die den Gegner überzeugen sollen, fördert nicht selten unglaubliche linguistische Verrenkungen zutage.

Wie schon weiter oben angedeutet, heißt der zentrale Topos „Sprachpolizei“, die Vorkommenshäufigkeit ist v. a. auf der Ebene der Laien, d. h. in den online-Leserkommentaren zu den Artikeln und in den Twittermeldungen, immens. Es findet sich zwar eine entsprechende Einstellung auch auf den anderen beiden Ebenen ${ }^{17}$, aber eben viel seltener und etwas gemäßigter, die Stichwörter lauten meistens „Zensur“ oder „politisch korrekt“. Die Angst vor der Diktatur der Sprachwächter scheint groß zu sein: Sie verfügt über alle entsprechenden Machtinstrumente wie Einflussnahme, Bevormundung, Kontrolle, Manipulation, Tabuisierung, Zensur, Verbannung, sie überwacht die Sprache und mittels der Sprache auch das Denken. Nicht zufällig stößt man auch häufig auf Hinweise auf Orwell, sei es als explizite Erwähnung („Orwell grüßt mit der Umformung der Wahrheit“) oder als Anspielung („Newspeak“, „Neusprech“). Oft hat die Diktatur auch einen Namen: politische Korrektheit, auch in der englischen Variante political correctness oder Abkürzung PC gängig. Als „politisch korrekt“ will meistens niemand bezeichnet

17 Die einzelnen Positionen (Akteure) des sprachkritischen Diskurses könnte man vielleicht folgendermaßen definieren: Erstens die „Experten“: in diesem Beitrag werden jedoch lediglich die Stimmen berücksichtigt, die auch über die Fachkreise hinaus präsent sind, d. h. die medialen Auftritte von Linguisten, hier vertreten durch die Pressemitteilungen der Jury der sprachkritischen Aktion „Unwort des Jahres“, mehrere Interviews mit der Sprachwissenschaftlerin Elisabeth Wehling und der Blog des Sprachwissenschaftlers Anatol Stefanowitsch. Die zweite Ebene ist die der „Vermittler“ - hier vertreten durch die großen überregionalen Zeitungen, siehe einzelne Teilkorpora, und schließlich drittens die „Stimme des Volkes“: in dem dieser Untersuchung zugrundeliegenden Korpus hierzu stellvertretend die Leserkommentare zu Zeitungsartikeln zum Thema und Beiträge in sozialen Medien, v. a. Twitter. Diese Unterteilung in drei Akteursebenen mag insofern sinnvoll sein, als für diese Gruppen bestimmte Diskurspositionen charakteristisch und mit der Expert-Laie-Stratifizierung teilweise erklärbar sind; man muss sich allerdings auch der Künstlichkeit einer solchen Kategorisierung bewusst sein, denn die einzelnen Ebenen durchdringen sich häufig. Auch Experten schreiben Leserkommentare und teilen bei Twitter, und unter den linguistischen Laien finden sich nicht selten solche, die mit den Experten die Einstellung zur Sprache teilen. 
werden, denn es gilt hierzulande eher als extremer Auswuchs mit fast ausschließlich negativen Konnotationen von steril, reserviert, steif.

„Und diese political correctness kann man sehr gut beschreiben (...): Sie setzt sich zusammen aus politischem Moralismus, aus einer Art Sprachhygiene, in einer Menge von Sprachtabus und darüber hinaus auch durchaus eine Art puritanischer lustfeindlicher Haltung." ${ }^{18}$

Dies sind also die beiden Extrempositionen, hinter denen sich die Auffassung von Sprache als „Handlung“ bzw. als „ausbleibende Handlung“ verbirgt. Diese Positionen und die mit ihnen verbundenen Topoi und argumentativen Strategien durchziehen alle hier behandelten Teildiskurse.

Bevor ich im Folgenden auf die Spezifika der beiden hier vertretenen sprachkritischen Debatten eingehe und deren Auslöser kurz skizziere, möchte ich sie in einen allgemeineren Rahmen einbetten - als ein solcher könnte hier die medial wirksamste sprachkritische Aktion „Unwort des Jahres“ fungieren, unter deren Wahlergebnissen sich zahlreiche weitere Beispiele für diese kritikwürdigen Strategien finden. Die Initiative möchte laut eigener Zielsetzung „auf öffentliche Formen des Sprachgebrauchs aufmerksam machen und dadurch das Sprachbewusstsein und die Sprachsensibilität in der Bevölkerung fördern.“19 Die Aktion „Unwort“ stößt auf starke Resonanz, in nahezu allen regionalen und überregionalen Zeitungen wird über die Wahl berichtet. Die Meldungen über die Wahl sind stark referierend und tragen inhaltlich sehr wenig zur Diskussion bei, aber die mediale Präsenz der sprachkritischen Aktion ist überwältigend. Zur Analyse werden daher einige ausgewählte stärker sprachreflexive Texte herangezogen. Im Rahmen dieses Aufsatzes sind v. a. die beiden Bereiche belastete Wörter und gefährliche Metaphern interessant.

Aus dem ersten Bereich könnten z. B. noch diese von der Jury kritisierten Ausdrücke genannt werden: Lügenpresse, entartet, ausländerfrei sowie das neueste Unwort von 2016 - Volksverräter. Bei den letzten beiden rügt die Jury in ihrer Begründung die pauschale Diffamierung mit Hilfe eines geschichtlich aufgeladenen Ausdrucks, der in der Vergangenheit als Schlagwort und Kampfbegriff geprägt wurde und nun erneut eingesetzt wird, um Debatten mit einem Schlag zu beenden ohne sie differenziert zu führen. Die zweite Gruppe, die auch den Lawinen-Vergleich einschließen würde, könnte man als "gefährliche Metaphern“ bezeichnen - dies sind Übertragungen und Überblendungen, die unauffällig gefährliche Analogien konstruieren, beispielsweise Flüchtlingsbekämpfung oder Rentnerschwemme. Kritikwürdig ist hier vor allem das hervorgerufene Bild eines Naturereignisses, das Bedrohung und unvermeidliches Geschehen suggeriert. In der Begründung der Jury wird explizit noch ein anderes Beispiel erwähnt, die Asylantenflut.

Im Folgenden werden die konkreten Ereignisse skizziert, die in der zweiten Hälfte des Jahres 2015 die Wellen der Sprachkritik höher schlagen ließen. Am 31. 8. 2015 hat

18 Interview mit dem Philosophen und Medienwissenschaftler Norbert Bolz im DeutschlandRadio Kultur: http://www.deutschlandradiokultur.de/gutmenschen-eben-mal-die-welt-retten.976.de.html?dram:article_ id=294305, letzter Zugriff am 14. 3. 2017.

19 http://www.unwortdesjahres.net/index.php?id=2 
die ARD die allwöchentliche Ausgabe der politischen Talkshow hart aber fair mit dem Titel „800000 Flüchtlinge - schafft Deutschland das?“ ausgestrahlt. Der bayerische Innenminister Joachim Herrmann wollte den Schlagersänger Roberto Blanco als Beispiel gelungener Integration herausstellen und sagte: „Roberto Blanco war immer ein wunderbarer Neger, der den meisten Deutschen wunderbar gefallen hat“. Diese saloppe Äußerung zog eine heftige sprachkritische Diskussion über den Tabu-Status des Wortes Neger nach sich, in der Herrmann natürlich ein relativ billiges Ziel abgab, gerade auch weil viele Kommentatoren es offenbar für überflüssig hielten, den Kontext der Äußerung mit zu berücksichtigen. Darauf verwies der bayerische Minister dann am folgenden Tag, als er zu seiner Entschuldigung daran erinnerte, dass er mit seinem Satz auf diesen Einspieler eines Zuschauers reagierte. Laut Herrmann also ein Zitat (das allerdings erst etwa vier Minuten später kam), laut seinen Kritikern eine kolossale Entgleisung, die weder durch Kontext noch durch die vorgebliche Geläufigkeit des Ausdrucks im bayerischen Dialekt zu rechtfertigen ist. Das Wort ist seit Jahren tabuisiert und nicht selten nur noch in der Form „N-Wort“ anzutreffen. Die N-Wort-Debatte ist zu diesem Zeitpunkt nicht zum ersten Mal geführt worden. Drei Jahre zuvor entbrannte eine heiße Diskussion darüber, ob der Negerkönig in Pippi Langstrumpf und das Negerlein in der Kleinen Hexe bleiben dürfen oder ob sie gestrichen/ersetzt werden sollen. Diese Diskussion verlief zwar unter etwas anderen Vorzeichen, denn es ging dort nicht um einen offensichtlichen und aktuellen Tabubruch, sondern um das Ausmaß an Schädlichkeit dieses Wortes bzw. um seine Verankerung in einer anderen Epoche, eine Art Zeitkolorit. Es scheint mir trotzdem angebracht, es hier zumindest zu erwähnen, da einige der Argumente alle hier untersuchten Debatten durchziehen. Angestoßen wurde die Diskussion durch das Interview mit der damaligen Familienministerin Kristina Schröder ${ }^{20}$ und ihre Antwort auf die Frage, wie sie selbst mit der diskriminierenden Sprache mancher Kinderbücher umgehen würde: „Ich werde synchron übersetzen, um mein Kind davor zu bewahren, solche Ausdrücke zu übernehmen. Auch ohne böse Absicht können Worte ja Schaden anrichten. Wenn ein Kind älter ist, würde ich dann erklären, was das Wort „Neger“ für eine Geschichte hat und dass es verletzend ist, das Wort zu verwenden.“ Man könnte meinen, das sei eigentlich eine harmlose Antwort (bedenkt man zudem, dass der Negerkönig bereits seit 2009 in den neuen Ausgaben von Pippi Langstrumpf durch den Südsee-König ersetzt wird), aber die Reaktionen waren zum Teil heftig. Es seien hier nur einige der Schlagzeilen angeführt:

\section{Kristina Schröder zensiert beim Vorlesen den „Negerkönig“"21 \\ Vorlese-Ratschläge für Eltern: Ministerin Schröder schafft den „Negerkönig“ a ${ }^{22}$ \\ Kristina Schröders Vorlesetechnik: (...) Pippi Langstrumpf nur nach Übersetzung ${ }^{23}$}

20 http://www.zeit.de/2012/52/Kristina-Schroeder-Interview, letzter Zugriff am 14. 3. 2017.

21 https://www.welt.de/politik/deutschland/article112098115/Schroeder-zensiert-beim-Lesen-den-Negerkoenig.html, letzter Zugriff am 14. 3. 2017.

22 http://www.spiegel.de/kultur/literatur/kristina-schroeder-liest-nicht-negerkoenig-vor-bei-pippilangstrumpf-a-873563.html, letzter Zugriff am 14. 3. 2017.

23 http://www.sueddeutsche.de/panorama/kristina-schroeders-vorlesetechnik-heihopp-was-wird-das-fuerein-leben-1.1554143, letzter Zugriff am 10. 3. 2017. 
Die kleine Hexenjagd. Aus Kinderbuch-Klassikern sollen Wörter gestrichen werden, die nicht mehr politisch korrekt sind. Das ist gut gemeint, aber ein Vergehen an der Literatur ${ }^{24}$

Political Correctness: Warum wir uns bevormundet fühlen ${ }^{25}$

Kinderbücher: Der Neger bleibt ein Neger. Die preisgekrönte Kinderbuchautorin Christine Nöstlinger will keine Wörter verhaften, um üble Gesinnung zu bekämpfen ${ }^{26}$

Es muss gerechterweise angemerkt werden, dass die meisten den unten angeführten Schlagzeilen zugehörigen Texte sich doch einigermaßen differenziert mit dem Thema auseinandersetzen und nicht so eindeutig in die durch die Headlines eingeschlagene Richtung gehen, ja ihre Argumentation in vielen Fällen sogar entgegengesetzt läuft trotzdem gilt: Sollten die Überschriften auch nur zitierend auf die in der öffentlichen Diskussion vorherrschende Meinung anspielen, manifestiert sich darin eine bestimmte Diskurs-Position. Bereits anhand der Überschriften lässt sich der bereits genannte wiederkehrende Haupttopos ausmachen: die „Sprachpolizei“ mit all den üblichen lexikalischen Varianten Zensur, (moralisch überlegene) Tugendwächter, Pfad der Gerechten, Hexenjagd, Tugendterror, Verhaftungen im Auftrag einer gerechten Sache, Bevormundung. In der N-Wort- und der Kinderbuch-Debatte geht es also um klar pejorative und verletzende Ausdrücke, die im breiten Konsens verpönt sind - die dazugehörige Debatte dreht sich dann eher um die Zulässigkeit bzw. Grenzen der moralisierenden Aufsicht über die (vermeintlichen) Tabubrüche und um die „sprachpolizeiliche“ Strenge und Kompromisslosigkeit bei der Ahndung derselben.

Knapp zwei Monate nach dem Neger-Eklat sorgt ein anderer Minister für Empörung. Wolfgang Schäuble verglich den massiven Flüchtlingsandrang mit einer Lawine und spielte diese Metapher gedanklich noch weiter durch:

„Lawinen kann man auslösen, wenn irgendein etwas unvorsichtiger Skifahrer an den Hang geht und ein bisschen Schnee bewegt. (...) Ob wir schon in dem Stadium sind, wo die Lawine im Tal unten angekommen ist, oder ob wir in dem Stadium im oberen Ende des Hanges sind, weiß ich nicht. “27

Es wird gerade im Zusammenhang mit dem Flüchtlingsdiskurs immer wieder darauf aufmerksam gemacht, dass Metaphern aus dem Bereich der Naturkatastrophen (eben Lawine, Flut, Welle, Strom) eine heikle Denkfigur darstellen, denn wenn man Flüchtlinge als Gefahr konzeptualisiert, die es „in einer Art Notwehr“ abzuwehren gilt, bzw. als „Masse“, deren einzelne Bestandteile nicht mehr sichtbar sind, sinkt die Hilfsbereitschaft. Die Perspektivierung und Konzeptualisierung der Welt mittels Sprache hat Auswirkungen

24 http://www.zeit.de/2013/04/Kinderbuch-Sprache-Politisch-Korrekt, letzter Zugriff am 14. 3. 2017.

25 http://www.zeit.de/kultur/literatur/2013-01/Political-Correctness-Essay, letzter Zugriff am 14. 3. 2017.

26 http://www.zeit.de/2013/05/Kinderbuecher-Sprache-Political-Correctness-Christine-Noestlinger, letzter Zugriff am 14. 3. 2017.

27 Zitiert wurde diese Äußerung selbstverständlich in allen überregionalen Medien, hier beispielsweise https://www.welt.de/politik/deutschland/article148748166/Schaeuble-warnt-in-Fluechtlingskrise-vor-Lawine. html, letzter Zugriff am 14. 3. 2017. 
auf unser Handeln. In diesem Kontext sei auf das grundlegende Werk von LAKOFF und JOHNSON Metaphors we live by bzw. in deutscher Übersetzung Das Leben in Metaphern verwiesen $^{28}$, das zwar nirgendwo in den hier untersuchten Debatten zitiert wird, dessen Hauptannahmen allerdings eine zentrale Rolle im gegenwärtigen sprachkritischen Diskurs spielen. Die Autoren gehen davon aus, dass „unsere Art zu denken, unser Erleben und unser Alltagshandeln weitgehend eine Sache der Metapher“29 sei, denn Metaphern sind nicht entgegen dem traditionellen Verständnis bloße Ornamente oder rhetorische Gesten, sondern Konzepte, die unsere Wahrnehmung strukturieren. Es handelt sich also um „konzeptuelle“ Metaphern, die eine kognitive Funktion haben und über ein hohes Implikationspotenzial verfügen. An dem berühmten Beispiel der konzeptuellen Metapher „Argumentieren ist Krieg“ (die sich in unzähligen Ausdrücken niederschlägt: Standpunkte angreifen, verteidigen, ins Schwarze treffen, Argumente abschmettern usw.) wird das zentrale Prinzip veranschaulicht: immer werden bestimmte Aspekte des jeweiligen Sachverhalts hervorgehoben und andere ausgeblendet ${ }^{30}$ - das zitierte Beispiel lässt also die Gegensätzlichkeit und Unvereinbarkeit der Standpunkte bzw. das Ringen um den Sieg hervortreten und durch die vielfältige sprachliche Ausgestaltung beinah als den einzig möglichen Entwurf erscheinen, die Aspekte der Kooperation hingegen werden als irrelevant, sekundär, randständig ausgeklammert.

Um zu der konkreten sprachkritischen Debatte zurückzukommen: Hier liegt der Schwerpunkt etwas anders und die Sache ist weniger eindeutig als bei der N-Wort-Debatte, denn es geht hier nicht mehr um ein eindeutiges Tabu-Wort, wo man sich mehrheitlich einig ist, dass es möglichst vermieden werden soll. Es geht zwar auch hier um Wörter, aber nicht um „belastete Wörter“, sondern um (un)erwünschte Assoziationen, die unsere Wahrnehmung und unser Denken strukturieren und in Folge dann unsere Haltungen in bestimmte Richtung lenken.

Im Jahr 2015 entschied sich die Jury der sprachkritischen Aktion „Unwort des Jahres“31 für das Wort bzw. Unwort Gutmensch. Die Begründung lautete: „Mit dem Vorwurf „Gutmensch“, „Gutbürger“ oder „Gutmenschentum“ werden Toleranz und Hilfsbereitschaft pauschal als naiv, dumm oder weltfremdes Helfersyndrom diffamiert. "32 Gerade in dieser Diskussion wird der Jury immer wieder vorgeworfen, sie treffe politische, nicht linguistische Entscheidungen, und tatsächlich werden nirgendwo die stilistische Auffälligkeit des Wortes und die hinter der Wortbildung verborgene, den Weg der Stigmatisierung leicht ebnende Strategie angesprochen. ${ }^{33}$ „Gutmensch“ als ein Adjektiv-Nomen-Kompositum

28 Lakoff, George/Johnson, Mark: Leben in Metaphern. Konstruktion und Gebrauch von Sprachbildern, Heidelberg: Carl Auer Verlag, 6. Auflage 2008.

29 Ebd., S. 11.

30 Ebd., Kap. 3: Metaphorische Systematik: Beleuchten und verbergen, S. 18-21; im engl. Original „highlighting and hiding“.

31 Zu den einzelnen Mitgliedern der Jury und den Auswahlkriterien siehe die Webseiten der Aktion: http:// www.unwortdesjahres.net/index.php?id=5, letzter Zugriff am 14. 3. 2017.

32 http://www.unwortdesjahres.net/fileadmin/unwort/download/pressemitteilung_unwort2015_neu.pdf

$33 \mathrm{Zu}$ Wortgeschichte sei verwiesen auf: http://gfds.de/gutmensch/, http://www.diss-duisburg. de/2011/11/das-stigma-gutmensch/ und http://muellers-lesezelt.de/aufsaetze/Gutmensch.pdf 
repräsentiert ein Wortbildungsmuster, das zwar weniger produktiv ist als die Nomen-Nomen- oder Verb-Nomen-Komposition, trotzdem aber zahlreiche Vertreter wie Alternativmedizin, Blindflug, Dunkelmänner oder Falschgeld aufweist. ${ }^{34}$ Semantisch gesehen sind alle Adjektiv-Nomen-Komposita nach dem gleichen Muster gebildet, die Attribuierung des Nomens durch das Adjektiv verläuft in gleicher Weise wie in äquivalenten Nominalphrasen: Blaukraut $=$ blaues Kraut, Billigmedikament $=$ billiges Medikament. Einige Adjektiv-Nomen-Komposita gehören allerdings diesem Attributmuster nicht an: Akutbett bedeutet nicht unmittelbar ,akutes Bett', Schnellstraße ist keine ,schnelle Straße‘. Eine Möglichkeit der Interpretation solcher Komposita ist die sog. Klammerform: Akut(fall) bett. Die erste Einheit wäre in dieser Zusammensetzung gar kein Adjektiv, sondern ein gekürztes Nomen, in dem die adjektivische Attribuierung „stimmt“: Akutfall (= akuter Fall) - Akutfallbett - Akutbett. ${ }^{35}$ Da „Gutmensch“ offenbar nicht dasselbe bedeutet wie „guter Mensch“, muss man sich fragen, ob es sich vielleicht auch um so eine „Klammerform“ handeln kann: Gutmensch ist ein Mensch, der Gutes - tut? will? vortäuscht? anderen diktiert? Gutmensch ist ein Kompositum, d.h. seine Bestandteile sind enger zusammengefügt - der Unterschied zu Konstruktionen mit attributivem Adjektiv ist die Benennungsfunktion bzw. Begriffsbildung, die der zugrundeliegenden Kompositionsregel inhärent ist (vgl. roter Wein vs. Rotwein, aber nicht Rotpullover).$^{36}$ Grundlegend ist also die Verbindung mit einem Konzept. Gutmensch ist nicht einfach ein „guter Mensch“, sondern ein (neuer) Typus, eine verfestigte Bezeichnung für jemanden, „der in der Öffentlichkeit - oft demonstrativ - moralisch und politisch korrekt handelt und redet “37: Ein Gutmensch ist ein „[naiver] Mensch, der sich in einer als unkritisch, übertrieben, nervtötend o. ä. empfundenen Weise im Sinne der Political Correctness verhält, sich für die Political Correctness einsetzt. “38 In den durch die Wahl ausgelösten Debatten herrscht denn auch der Topos „selbsternannter (Tugend-)Wächter“, der die „Moralkeule“ schwenkt und sich allen anderen überlegen fühlt - denn die Gutmenschen werden kurzerhand mit den Sprachsensibilisierern gleichgesetzt.

Die höchsten Wellen hat das 2015 in einer Art Parallelaktion - der sprachkritischen Initiative des GfdS - gewählte „Wort des Jahres“ Flüchtling geschlagen. Im Gegensatz zu den gerügten Unwörtern sind allerdings die „gekürten“ Wörter und Wendungen (des Jahres) mit keinerlei Wertung verbunden. Dies bezeugt auch die Formulierung in der Mitteilung der Jury: Der Zankapfel in der sprachkritischen Debatte um das Wort des Jahres 2015 - nämlich das Suffix -ling - sei lediglich „sprachlich interessant“. Im Jahr

34 http://hypermedia.ids-mannheim.de/call/public/sysgram.ansicht?v_typ=d\&v_id=910

35 Ebd.

36 Hierzu näher ROTH, Tobias: Wortverbindungen und Verbindungen von Wörtern. Lexikografische und distributionelle Aspekte kombinatorischer Begriffsbildung zwischen Syntax und Morphologie. Tübingen: Francke, 2014, S. 151ff.

37 Definition im Online-Wortschatz-Informationssystem Deutsch (Portal für wissenschaftliche, korpusbasierte Lexikografie des Instituts für Deutsche Sprache): http://www.owid.de/nav/gehezu/gutmensch?module=ctx.all; letzter Zugriff am 14. 2. 2017.

38 Definition im DUDEN online: http://www.duden.de/suchen/dudenonline/gutmensch; letzter Zugriff am 14. 2. 2017. 
2015 wurde der Ausdruck Flüchtlinge zum Wort des Jahres gewählt - absichtlich im Plural, denn dieser ist selbst ein Aspekt des Phänomens. Die Begründung der Jury lautet:

Das Substantiv steht nicht nur für das beherrschende Thema des Jahres, sondern ist auch sprachlich interessant. Gebildet aus dem Verb flüchten und dem Ableitungssuffix -ling (>Person, die durch eine Eigenschaft oder ein Merkmal charakterisiert ist<), klingt Flüchtling für sprachsensible Ohren tendenziell abschätzig: Analoge Bildungen wie Eindringling, Emporkömmling oder Schreiberling sind negativ konnotiert, andere wie Prüfling, Lehrling, Findling, Sträfling oder Schützling haben eine deutlich passive Komponente. Neuerdings ist daher öfters alternativ von Geflüchteten die Rede. Ob sich dieser Ausdruck im allgemeinen Sprachgebrauch durchsetzen wird, bleibt abzuwarten. ${ }^{39}$

Die Bezeichnung sei also „tendenziell abschätzig“ - in den Reaktionen auf die Wahl geht es dann vorwiegend um die Frage, wie viel Substanz diese Behauptung hat. In der eben zitierten Begründung der Jury werden zwei Aspekte der Kritikwürdigkeit genannt: die schlechte Gesellschaft der analogen (d. h. durch die -ling-Suffigierung entstandenen) Bildungen, deren pejorative Bedeutung „abfärbt“, sowie die durch den Ausdruck suggerierte Passivität. Diese beiden Kritikpunkte wurden durch die flächendeckende mediale Präsenz der Aktion „Wort des Jahres“ tausendfach reproduziert und dienen in den Kommentarbereichen der Zeitungen als die Hauptkontrastfolie für die z. T. heftigen Reaktionen auf die Wahl. Darüber hinaus gibt es noch zwei weitere Argumente dafür, dass das Wort Flüchtling bald als ein Unwort eingestuft werden könnte: zum einen das in allen sprachkritischen Debatten omnipräsente Genderproblem - es lässt sich keine feminine Form bilden - und die (zunehmende) Verwendung des Ausdrucks in Zusammensetzungen wie Flüchtlingskrise oder Flüchtlingsproblem.

Am interessantesten sind zweifellos die Ausführungen zu dem Suffix -ling: Zum einen könnten sie als eindrucksvoller Beweis für die Notwendigkeit linguistischer Analysen dienen, denn selten wird in einer dermaßen von Bauchgefühlen ${ }^{40}$ und subjektiven Empfindungen dominierten Debatte so heftig (und so falsch) mit Fachbegriffen hantiert.

„Man kann selbstverständlich Partizipien zu Substantiven machen, sollte aber darauf achten, dass die grammatikalische Funktion erhalten bleibt. Ein Partizip Perfekt hat in der Regel passivische Funktion: Ein Geretteter ist einer, der gerettet worden ist. Was wäre dann ein Geflüchteter? Die entsprechende Ersatzprobe wäre absurd!“41

In dem Ausdruck Flüchtling scheinen sich mehrere Strategien der Pejoration zu verschränken: im Lexem selbst angelegt (durch die Basis vermittelt oder aus der Funktion

39 http://gfds.de/wort-des-jahres-2015/, letzter Zugriff am 10. 3. 2017.

40 Ich habe noch einmal in mich hineingehorcht und konnte beim Wort „Flüchtling“ keine negative Konnotation entdecken. (tsp 2); „Die Wirkung von Sprache (...) ist nur zu erleben. Wie fühlt sich das Wort Flüchtling an? Was macht es mit mir? Was erzeugt es?" (stef 1 - 2015). Die Frage ist, inwieweit man sich auf ein „untrainiertes“ Gefühl verlassen kann, aber da ist man wieder bei der Forderung der Sensibilisierung.

41 http://www.sprachlog.de/2012/12/01/fluechtlinge-und-gefluechtete/, letzter Zugriff am 12. 12. 2016. 
des Suffixes resultierend) oder im Sprachgebrauch entstehend. Diese Überlappung sorgt für Verwirrungen und fördert zuweilen für solche an Sprachmagie grenzenden Vorschläge, man solle die Flucht ganz aus der Sprache verbannen, die Flüchtlinge nicht auf ihr Flüchtlingssein reduzieren, sondern von „Menschen, die zu anderen Menschen kommen"42 sprechen. Aber selbst Fachleute sind vor Widersprüchen nicht gefeit - die weiter oben bereits erwähnte Sprachwissenschaftlerin Elisabeth Wehling, die ein in diesem Kontext interessantes Buch zum „politischen Framing“"43 verfasst hat und wiederholt die Wortwahl in der aktuellen Flüchtlingsdebatte anprangert, behauptet:

„Allein schon das Wort Flüchtling. [...] Das ist ein Frame, der sich politisch gegen Flüchtlinge richtet. [...] Die Endung „-ling“ macht diese Menschen klein und wertet sie ab. [...] Außerdem ist „der“ Flüchtling männlich - und damit transportiert dieses Wort sehr viele männliche Merkmale: „Der“ Flüchtling ist eher stark als hilfsbedürftig, eher aggressiv als umgänglich. “44

Die sprachwissenschaftliche Analyse dieses umstrittenen Ausdrucks kann unter der spezifischen Perspektivierung nicht Gegenstand dieses Aufsatzes sein, verwiesen sei an dieser Stelle lediglich auf die sehr aufschlussreiche Arbeit von Heike Baeskow ${ }^{45}$. Zusammenfassend lässt sich festhalten, dass im heutigen Deutsch das Suffix -ling zur Bildung von folgenden Substantivgruppen dient: Nomina Patientis (Prüfling), Nomina Qualitatis (Schönling), bei denen meistens eine pejorative Modifizierung gegenüber dem Basiswort auftritt, Nomina Instrumenti (Fäustling), Personenbezeichnungen, die auf gleichaltrige Geschwister referieren und Numeralia als Basis aufweisen (Zwilling) sowie Nomina Agentis (Eindringling), die ebenfalls überwiegend pejorativ sind. Das Suffix hatte ursprünglich eine diminutive Funktion ohne negative Konnotationen - es bezeichnete junge oder kleine Tiere: Frischling (junges Wildschwein), Nestling (Raubvogel, der noch nicht fliegen kann), Schmetterling. Eine wichtige Rolle spielt im Falle dieses Suffixes die Sprachentwicklung, nämlich dessen zurückgegangene Produktivität, denn die meisten auf diese Weise gebildeten Substantive sind aus dem heutigen Wortschatz verschwunden: Durch die Exponiertheit kann der pejorative Kontext sichtbarer abfärben als bei einer Allerweltsendung wie beispielsweise -ung.

Wie oben bereits erwähnt, wurde zur Wahl des „Wortes des Jahres“ in den Kommentarbereichen der Zeitungen eine hitzige Debatte geführt, in der die „tendenzielle Abschätzigkeit“ die Hauptrolle spielt, die mit jeweils mehr oder weniger überzeugenden Beispielen entweder behauptet oder verworfen wird. Der zentrale Topos in dieser sprachlichen Flüchtlings-Debatte ist die Sprachpolizei, die zensiert, tabuisiert, maßregelt, lenkt, verbietet, erziehen will - aber vor allem überflüssig ist. Außerdem wird hier mehr

42 Leserkommentare zu: http://www.zeit.de/gesellschaft/zeitgeschehen/2015-12/fluechtlinge-wort-desjahres\#comments, letzter Zugriff am 14. 3. 2017.

43 WEHLING, Elisabeth: Politisches Framing. Wie eine Nation sich ihr Denken einredet - und daraus Politik macht. Köln: Herbert von Halem Verlag, 2016.

44 http://www.zeit.de/2016/10/sprache-manipulation-elisabeth-wehling, letzter Zugriff am 14. 3. 2017.

45 BAESKOW, Heike: Abgeleitete Personenbezeichnungen im Deutschen und Englischen. Berlin: de Gruyter, 2002, S. 500-536. 
als bei den anderen Teildiskursen explizit auf die große Verunsicherung und Absurdität der Alternativbezeichnungen verwiesen. Der Grund dafür dürfte der starke moralische Impetus der "Sprachpfleger“ sein, der in diesem Kontext - im starken Gegensatz zu den Diskussionen um „Neger“ und „Lawine“ - nur den wenigsten nachvollziehbar scheint:

„Also sorry, bisher fand ich „Prüfling“ völlig neutral [...] Aber nee, ich lerne gerade, dass ich das alles negativ besetzt finde. Bekomme ich gerade wirklich gesagt, welche Assoziationen zulässig sind?" 46

„Beim Problem „sensibler Sprachregelungen“ [...] beschleicht mich ein mulmiges Gefühl. [...] Ich habe manchmal den Eindruck, solche Texte (der politisch Korrekten) wollen mir unterschwellig sagen: „Wir sind reflektiert, sensibel, avantgardistisch, problemorientiert und aufgeklärt. Und du, wenn du nicht so schreibst wie wir, bist hinterwäldlerisch, rassistisch, chauvinistisch, dumm und brutal." 47

Der durch den Begriff der (Sprach-)Polizei aufgerufene Rahmen, um nochmals die Frame-Theorie zu bemühen, hält auch die gedankliche Entwicklung zum Polizeistaat und Diktatur bereit, und tatsächlich finden sich in vielen sprachkritischen Debatten Hinweise auf Orwell - die sehr emotional geladene Diskussion um den Ausdruck Flüchtling geht noch einen Schritt weiter und findet einen realen Vergleich: die „Wortergreifung“. Der Topos der Sprachpolizei ist - in einer moderateren Form natürlich - auch in den Texten seriöser Zeitungen zu finden, beispielsweise in den Interviews mit der bereits erwähnten Sprachwissenschaftlerin Elisabeth Wehling. Sie wird in jedem Gespräch mindesten einmal darauf angesprochen, ob der von ihr vorgeschlagene Sprachgebrauch nicht eine Sprachlenkung und zuletzt auch Manipulation ist - ihre Antwort lautet jedes Mal, die alleinige Absicht sei lediglich die Sensibilisierung. Man kann der sicher berechtigten Empfehlung nachkommen, nach jeder Zeitungslektüre über die Begriffsverwendungen nachzudenken, um sich über die vorherrschenden Gedankenmuster klar zu werden (es ist eigentlich eine Art alltägliche private Diskursanalyse), allerdings folgt eine ziemlich banale Erkenntnis daraus, nämlich dass Begriffe Einstellungen ausdrücken, was wohl niemand in Frage stellen würde. Es scheint hier aber im Hintergrund noch eine stillschweigende Annahme mitzuspielen: Obwohl man vorgibt, nur auf die Sprache achten und sensibilisieren zu wollen, ist man keineswegs neutral, sondern schlägt sich auf der Seite der „Gerechtigkeit“, und obwohl man immer wieder auf die „Mehrstimmigkeit“ als das Hauptmerkmal der Demokratie pocht, wird abweichender Sprachgebrauch - nicht nur in seiner extremen Form - disqualifiziert.

Es werden auch Zweifel an der Unparteilichkeit der Sensibilisierungsmission geäußert: Es sollen nicht negativ konnotierte oder pejorative Ausdrücke durch neutrale ersetzt werden, sondern das Ziel ist Erziehung zum richtigen Denken, positive (also: politisch korrekte) Konnotationen sollen in den Köpfen Wurzeln schlagen. Die Sprachlenkung der PC zeichnet sich durch eben diese Überzeugung von (All-)Macht der Sprache

46 http://www.spiegel.de/kultur/gesellschaft/fluechtlinge-ist-wort-des-jahres-2015-a-1067253.html, letzter Zugriff am 14. 3. 2017.

47 http://www.sprachlog.de/2012/12/01/fluechtlinge-und-gefluechtete/, letzter Zugriff am 14. 3. 2015. 
aus: Die sich in der Sprache manifestierenden alten Herrschaftsstrukturen sollten mittels neuer Sprachverwendungen aufgebrochen und gesellschaftliche Verhältnisse mittels Sprache verändert werden. Am deutlichsten manifestiert sich diese Einstellung bei den Alternativvorschlägen zu Flüchtling. Bei Zufluchtsuchenden würde der zu schaffende Ort des Schutzes, also die Gegenwart und unsere Aufgabe betont, bei Geflüchteten beziehe uns das Wort als Gastgeber und Notlinderer mit ein und nehme uns in die Pflicht, bei Vertriebenen versucht man, eine noch stärkere Empathie und Erinnerung an das Schicksal der vor 70 Jahren Vertriebenen zu erreichen. An der aufgeregten Diskussion, die sich um die „tendenzielle Abschätzigkeit“ des Wortes Flüchtling dreht, wird aber auch die um sich greifende Unsicherheit im Sprachgebrauch, die Kehrseite der Sensibilisierung deutlich.

„Ich mag das Wort „Geflüchtete“ auch noch aus einem anderen Grund. Es erinnert mich an das Wort „Gegangen“. Es zeigt, dass nicht alle Menschen, die fliehen, dies auch freiwillig tun.“ „Bei „Geflüchtete“ assoziiere ich bestenfalls Leute, die „irgendwie abgehauen sind“. Es schmeckt mir wie Ersatz-Kaffee.“

„Mir scheint die Gegenüberstellung von „Flüchtling“ und „Geflüchteter“ nicht ganz zu passen. Ein „Flüchtling“ ist doch jemand, der noch auf der Flucht ist - also jemand, dessen Flucht noch nicht abgeschlossen ist. Ein „Geflüchteter“, wie auch bei den „Gelandeten“, beschreibt jemand, der auf der Flucht war und es nun nicht mehr ist.“

„ich habe sehr oft ,flüchtlingsaktivist_innen“ benutzt. [...] keine ahnung, ob das eine gute lösung ist...? leider würde das mit ,geflüchteten“ komisch klingen: geflüchteten-aktivist_innen oder mensch müsste schreiben: die geflüchteten aktivist_innen."48

Der Beitrag widmete sich einzelnen Strängen der aktuellen sprachkritischen Debatte, die im Zuge der Flüchtlingskrise einen neuen Ansporn erhielt. Das Ziel war zu zeigen, um welche zentralen Topoi sich die Diskussion dreht und welche Auffassungen von Sprache sich dahinter verbergen. Es wurden auch die beiden sprachreflexiven Aktionen, die medial sehr präsent sind und in der Öffentlichkeit auf sehr starke (und nicht nur positive) Resonanz stoßen, das „Wort“ und „Unwort des Jahres“ herangezogen, um einige der kritikwürdigen Strategien näher betrachten zu können.

\section{Zitierte Quellen}

BAESKOW, Heike (2002): Abgeleitete Personenbezeichnungen im Deutschen und Englischen. Berlin: de Gruyter.

DIECKMANN, Walther (1984): Herrschaft durch Sprache durch Herrschaft über Begriffe. In: STÖTZEL, Georg (Hrsg.): Germanistik - Forschungsstand und Perspektiven. Vorträge des Deutschen Germanistentages 1984. Berlin: de Gruyter.

48 Alle Kommentare zum: http://www.sprachlog.de/2012/12/01/fluechtlinge-und-gefluechtete/, letzter Zugriff am 14. 3. 2017. 
GEISSLER, Heiner (1984): Sprache und Politik. Kampf um Begriffe. In: STÖTZEL, Georg (Hrsg.): Germanistik - Forschungsstand und Perspektiven. Vorträge des Deutschen Germanistentages 1984. Berlin: de Gruyter.

GLOTZ, Peter (1984): Die Rückkehr der Mythen in die Sprache der Politik. In: STÖTZEL, Georg (Hrsg.): Germanistik - Forschungsstand und Perspektiven. Vorträge des Deutschen Germanistentages 1984. Berlin: de Gruyter.

KILIAN, Jörg (Hrsg.) (2005): Sprache und Politik. Deutsch im demokratischen Staat. Mannheim: Dudenverl.

KLEIN, Josef (1991): Kann man „Begriffe besetzen“? Zur linguistischen Differenzierung einer plakativen politischen Metapher. In: LIEDTKE, Frank/WENGELER, Martin/BÖKE, Karin (Hrsg.): Begriffe besetzen. Strategien des Sprachgebrauchs in der Politik. Opladen: Westdt. Verl.

LAKOFF, George/JOHNSON, Mark (2008): Leben in Metaphern. Konstruktion und Gebrauch von Sprachbildern, Heidelberg: Carl Auer Verlag, 6. Auflage.

NIEHR, Thomas (2014): Einführung in die Politolinguistik. Gegenstände und Methoden. Göttingen: Vandenhoeck \& Ruprecht.

ROTH, Tobias (2014): Wortverbindungen und Verbindungen von Wörtern. Lexikografische und distributionelle Aspekte kombinatorischer Begriffsbildung zwischen Syntax und Morphologie. Tübingen: Francke.

WEHLING, Elisabeth (2016): Politisches Framing. Wie eine Nation sich ihr Denken einredet - und daraus Politik macht. Köln: Herbert von Halem Verlag.

\section{Teilkorpus zur N-Wort-Debatte}

http://www.faz.net/aktuell/feuilleton/tv-kritik-hart-aber-fair-wir-koennen-das-schaffen-13779138. html

https://www.welt.de/vermischtes/article145842397/CSU-Minister-nennt-Roberto-Blancowunderbaren-Neger.html

http://www.sueddeutsche.de/kultur/sprache-und-rassismus-worte-vermeiden-die-das-leiderneuern-1.2629592

http://www.zeit.de/kultur/2015-09/rassismus-neger-roberto-blanco-10nach8

http://www.taz.de/!5224306/

http://www.n-tv.de/politik/politik_kommentare/So-schwer-ist-das-eigentlich-nicht-article15835391.html

http://www.sueddeutsche.de/politik/ihr-forum-wunderbarer-neger-ist-herrmanns-aussage-ein-ausrutscher-oder-kalkuel-1.2629539

\section{Teilkorpus zur Kinderbuch-Debatte}

http://www.zeit.de/2012/52/Kristina-Schroeder-Interview

https://www.welt.de/politik/deutschland/article112098115/Schroeder-zensiert-beim-Lesenden-Negerkoenig.html

https://www.welt.de/debatte/henryk-m-broder/article112114240/Frau-Gott-laesst-sich-nicht-allesgefallen.html 
http://www.spiegel.de/kultur/literatur/kristina-schroeder-liest-nicht-negerkoenig-vor-bei-pippilangstrumpf-a-873563.html

http://www.sueddeutsche.de/panorama/kristina-schroeders-vorlesetechnik-heihopp-was-wirddas-fuer-ein-leben-1.1554143

http://www.zeit.de/2013/04/Kinderbuch-Sprache-Politisch-Korrekt

http://www.zeit.de/2013/05/Kinderbuecher-Sprache-Political-Correctness-Christine-Noestlinger http://www.zeit.de/kultur/literatur/2013-01/Political-Correctness-Essay

\section{Teilkorpus zum Lawinen-Vergleich}

https://www.welt.de/politik/deutschland/article148761529/Maas-wirft-Schaeuble-verbale-Brandstiftung-vor.html

http://www.spiegel.de/politik/deutschland/wolfgang-schaeuble-lawinen-vergleich-empoertdie-spd-a-1062454.html

http://www.tagesspiegel.de/politik/schaeubles-vergleich-zur-fluechtlingskrise-lawine-falschesbild-richtiger-appell/12577590.html

http:/ / www.n-tv.de/politik/SPD-greift-Schaeuble-nach-Lawinen-Zitat-an-article16338526.html

https://www.welt.de/politik/deutschland/article148748166/Schaeuble-warnt-in-Fluechtlingskrisevor-Lawine.html

http://www.faz.net/aktuell/politik/fluechtlingskrise/wolfgang-schaeuble-warnt-vor-lawine-influechtlingskrise-13907768.html

http:/ / www.faz.net/aktuell/politik/fluechtlingskrise-schaeuble-verteidigt-vergleich-mitlawine-13939778.html

http:/ / www.sueddeutsche.de/news/politik/migration-von-lawinen-und-ausloesern-schaeublestichelt-dpa.urn-newsml-dpa-com-20090101-151112-99-07414

http://www.sueddeutsche.de/news/politik/migration-fluechtlingskrise-kritik-an-schaeubleslawinen-vergleich-dpa.urn-newsml-dpa-com-20090101-151112-99-01094

http://www.spiegel.de/politik/deutschland/wolfgang-schaeuble-vergleicht-fluechtlingsbewegungmit-lawine-a-1062373.html

http://www.spiegel.de/politik/deutschland/wolfgang-schaeuble-zu-fluechtlingen-die-lawinen-entgleisung-kommentar-a-1062402.html

https:/ / twitter.com/search?q=\%23sch\%C3\%A4uble\%20lawine\&src=typd\&lang=de

\section{Teilkorpus zum „Unwort des Jahres"}

http:/ / www.tagesspiegel.de/weltspiegel/unwort-des-jahres-2015-gutmenschen-das-sind-immer-dieanderen/12820480.html

http://www.faz.net/aktuell/gesellschaft/unwort-des-jahres-der-gutmensch-ist-zurueck-14010334. html

https://www.welt.de/kultur/article138678946/Wer-Gutmensch-sagt-verdient-sich-seinen-Shitstorm.html

http://www.zeit.de/gesellschaft/2015-04/altruismus-gutmensch-motive

http://www.deutschlandradiokultur.de/gutmenschen-eben-mal-die-welt-retten.976.de.html?dram:article_id=294305 
http://www.sprachlog.de/2016/01/12/unwort-des-jahres-2015-gutmensch/

http://www.zeit.de/kultur/2016-01/unwort-des-jahres-2015-gutmensch\#comments

http://www.sueddeutsche.de/kultur/ihr-forum-gutmensch-auch-ihr-unwort-des-jahres-1.2814630

\section{Teilkorpus zum „Wort des Jahres"}

http://www.srf.ch/news/international/fluechtlinge-oder-fluechtende-sprache-ist-politik http://www.zeit.de/2016/10/sprache-manipulation-elisabeth-wehling http://www.sueddeutsche.de/kultur/sprache-in-der-fluechtlingsdebatte-das-wort-fluechtling-richtet-schaden-an-1.2864820

http://www.sueddeutsche.de/muenchen/fuerstenfeldbruck/sz-interview-sprache-kann-verletzen-1.2782791

https://www.welt.de/kultur/article145550890/Warum-Fluechtlinge-jetzt-oft-Refugees-heissen. html

http://derstandard.at/2000022449906/Asylanten-Fluechtlinge-Refugees-und-Vertriebene-eineSprachkritik

http://www.derwesten.de/kultur/ist-fluechtling-auf-dem-weg-zum-schimpfwort-id11533257.html http://www.tagesspiegel.de/politik/politisch-korrekte-sprache-neue-worte-braucht-das-land/ 12776234.html

http://www.deutschlandradiokultur.de/gefluechtete-versus-asylanten-begriffe-druecken.1005. de.html?dram:article_id=330623

http://www.spiegel.de/kultur/gesellschaft/fluechtlinge-ist-wort-des-jahres-2015-a-1067253.html http://www.sprachlog.de/2015/12/12/fluechtlinge-zu-gefluechteten/ http://www.sprachlog.de/2012/12/01/fluechtlinge-und-gefluechtete/ http://www.zeit.de/gesellschaft/zeitgeschehen/2015-12/fluechtlinge-wort-des-jahres\#comments

Markéta Ederová, M.A. / mederova@ff.jcu.cz

Jihočeská univerzita v Českých Budějovicích, Filozofická fakulta, Ústav česko-německých areálových studií a germanistiky

Branišovská 31a, 37005 České Budějovice, CZ 
